\title{
Atrioesophageal fistula after epicardial ablation for atrial fibrillation
}

\author{
Tijs Bringmans, MD, ${ }^{\mathrm{a}, \mathrm{b}}$ Anton Verrijcken, MD, ${ }^{\mathrm{b}}$ Mark La Meir, MD, PhD, ${ }^{\mathrm{c}}$ and Filip Rega, MD, PhD, ${ }^{\mathrm{d}}$ \\ Leuven, Turnhout, and Brussels, Belgium
}

\footnotetext{
From the Departments of ${ }^{\mathrm{a}}$ Internal Medicine and ${ }^{\mathrm{d}}$ Cardiovascular Surgery, University Hospitals Leuven, Leuven; ${ }^{\mathrm{b}}$ Department of Cardiology, General Hospital Turnhout, Turnhout; and ${ }^{\mathrm{c}}$ Department of Cardiovascular Surgery, University Hospitals Brussels, Brussels, Belgium.

Disclosures: M.L.M. has received consulting fees from AtriCure $(>\$ 10,000)$. F.R. has received consulting fees from AtriCure and Medtronic $(<\$ 10,000)$; has received lecture fees from speaking at the invitation of Medtronic, LivaNova, and AtriCure $(<\$ 10,000)$ and currently receives grant support from Medtronic. All other authors have nothing to disclose with regard to commercial support.

Received for publication April 1, 2017; revisions received July 25, 2017; accepted for publication Aug 23, 2017; available ahead of print Sept 22, 2017.

Address for reprints: Filip Rega, MD, PhD, Herestraat 49, B-3000, Leuven, Belgium (E-mail: filip.rega@ uzleuven.be).

J Thorac Cardiovasc Surg 2018;155:e19-21

$0022-5223 / \$ 36.00$

Copyright (c) 2017 by The American Association for Thoracic Surgery
}

http://dx.doi.org/10.1016/j.jtcvs.2017.08.069

Video clip is available online.

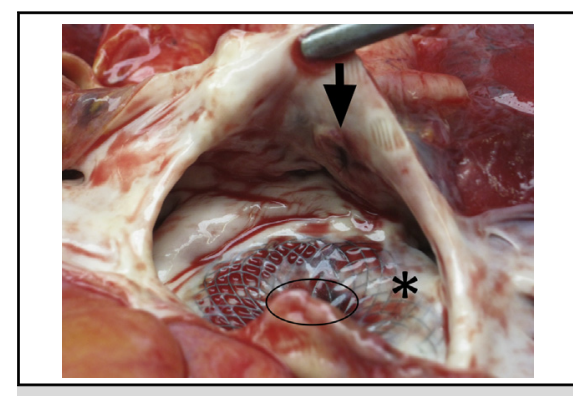

Autopsy shows spacing of fistula orifice (arrow) and atrial septal occluder (asterisk).

Central Message

An atrial septal occluder might be the cause of an atrioesophageal fistula after epicardial radiofrequency ablation for atrial fibrillation.

See Editorial Commentary page e23.
An atrioesophageal fistula (AEF) is a rare yet devastating complication after atrial fibrillation (AF) ablation. Clinical features are relatively straightforward, but clinical awareness remains low among clinicians and surgeons. There are therefore delays in presentation, diagnosis, and intervention, all contributing to the high mortality associated with AEF. In the case presented here, we believe that the presence of an atrial septal occluder contributed to AEF formation after epicardial radiofrequency ablation for AF.

\section{CLINICAL SUMMARY}

A 49-year-old Asian woman with drug-refractory AF and a previously unsuccessful percutaneous ablation procedure underwent surgical bipolar epicardial radiofrequency ablation. Other relevant medical history included percutaneous coronary intervention for anterior myocardial infarction, with stenting of the proximal left anterior descending coronary artery and percutaneous closure of an atrial septal defect type 2 with a 30-mm St. Jude Amplatzer atrial septal occluder (St. Jude Medical, LLC, St Paul, Minn) 10 years before presentation.

The procedure was performed through a bilateral thoracoscopy (Video 1). Electrical isolation of the right and left pulmonary veins was achieved with a bipolar, bidirectional radiofrequency clamp (Isolator Synergy Ablation Clamp; AtriCure, Inc, Mason, Ohio). Six applications were performed on each pair of pulmonary veins. A roof and inferior line was created with a bipolar unidirectional radiofrequency rail (Coolrail Linear Pen; AtriCure, Inc, Mason, Ohio). A 30-second cooling period with simultaneous quenching of the device, the ablated tissue, and the surrounding tissues with saline solution was applied to allow adequate cooling. The transesophageal echocardiographic probe was retracted before ablation began. An AtriClip (size 35; AtriCure) was placed at the base of the left atrial appendage at the end of the procedure.

Thirty days after the epicardial procedure, the patient was seen with progressive neurologic deterioration, with mutism and irregular stereotypical movements of all limbs in a lateralized athetotic pattern with more movement on the left, most consistent with a nonconvulsive status epilepticus for which antiepileptic therapy and empirical antiviral therapy was initiated. Full neuroprotective sedation and ventilation were applied. An inflammatory blood panel with elevated liver enzymes evolved. Severe lactic acidosis was present. Brain natriuretic peptide was markedly elevated (2098 ng/ L). A progressive anemia occurred, for which transfusion was applied. Multiple computed tomographic scans of the brain revealed no abnormalities. Electroencephalography showed no ictal activity. An apical implantable loop recorder made it difficult to evaluate cardiac function transthoracically. A transesophageal echocardiogram showed decreased left ventricular function (left ventricular ejection fraction of $35 \%$ ) with diffuse hypokinesia and anteroseptal akinesia, a 


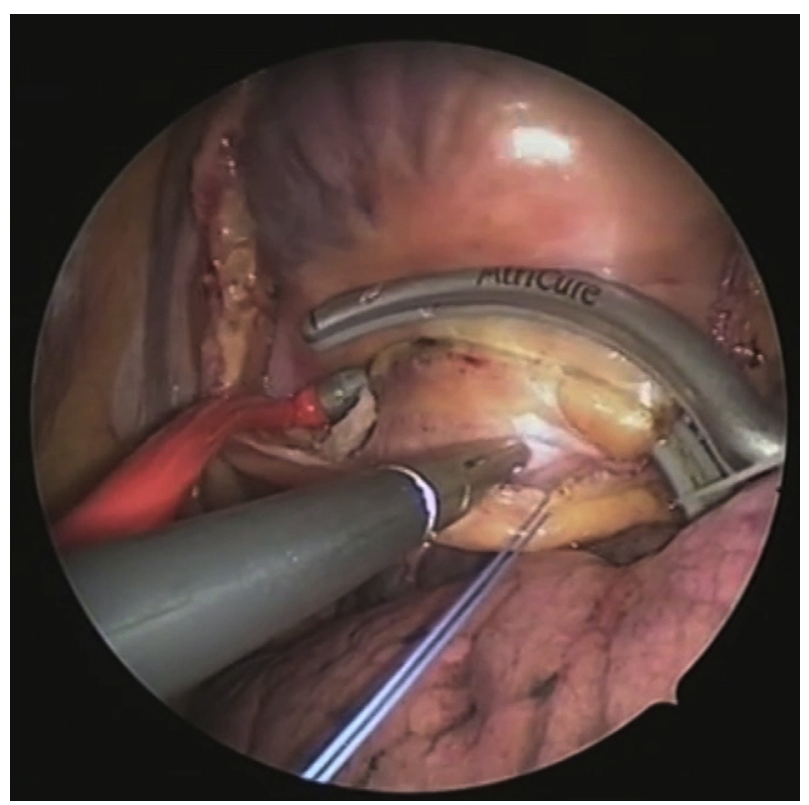

VIDEO 1. A similar thoracoscopic ablation procedure for atrial fibrillation is shown. The video illustrates the isolation of the pulmonary veins with the use of the AtriCure Coolrail Linear Pen and the AtriCure Synergy Isolator Clamps (AtriCure, Inc, Mason, Ohio). Video available at: http://www. jtcvsonline.org/article/S0022-5223(17)31831-7/fulltext.

restrictive filling pattern, minimal valve insufficiencies, and an interatrial closure device with correct positioning. No vegetation or shunt was visualized. A lumbar puncture sample drawn after cessation of rivaroxaban excluded pleocytosis and was culture negative. A fever developed. Blood cultures grew Streptococcus salivarius and Gemella species for which ceftriaxone and gentamicin were started. Episodes of AF with fast ventricular response occurred. A therapeutic dosage of low-molecular weight heparin was started after lumbar puncture.

On day 4, the patient was transferred to our surgical center for further diagnostic investigations. During the last $15 \mathrm{mi}-$ nutes of transport, hypotension developed, for which vasopressor therapy and crystalloid fluids were started. Shortly after the patient was placed in the cardiac intensive care unit, massive hematemesis with immediate hemodynamic instability occurred. Maximum efforts for cardiopulmonary resuscitation, reversal of anticoagulation and massive transfusion proved to be insufficient. After more than 1 hour, the resuscitation team decided to withdraw all further efforts, at which point the patient died of exsanguination. A postmortem study confirmed the presence of an AEF.

\section{DISCUSSION}

AEF is an increasingly recognized complication after ablation of AF in both percutaneous and surgical series. A typical clinical presentation consists of primary neurologic symptoms, ${ }^{1}$ caused by the capability of the esophagus to develop pressures 10 times greater than atrial pressures,

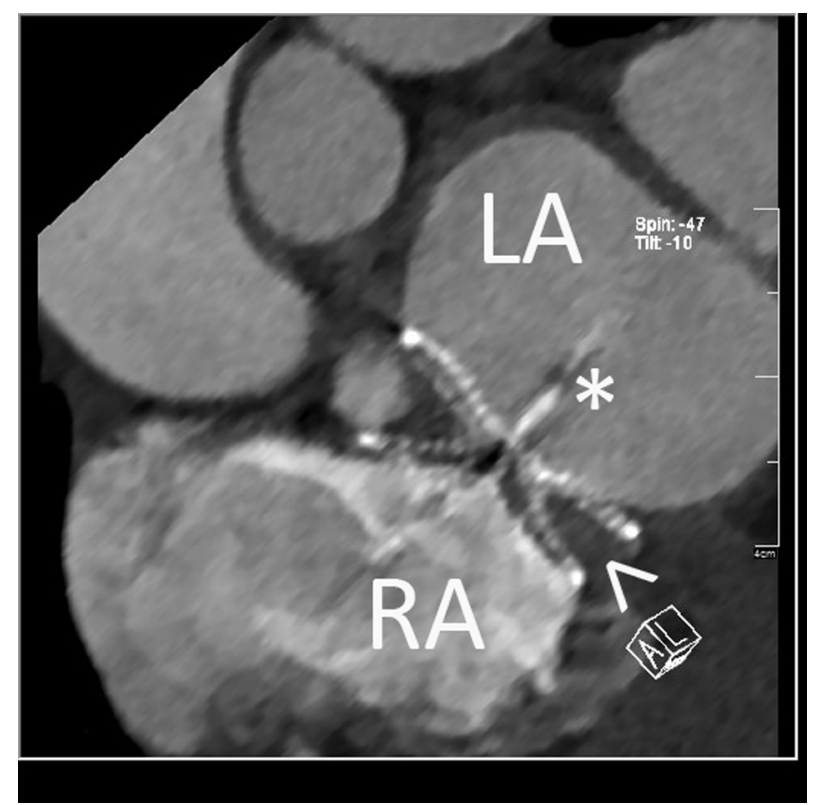

FIGURE 1. Computed tomographic image demonstrates the platinum left atrial (LA) marker band (asterisk) of the atrial septal occluder (arrowhead) in the left atrial lumen. $R A$, Right atrium.

which allows air, food, and bacterial emboli to enter the heart and cause embolization in the brain. Gastrointestinal blood loss is therefore not a typical presenting symptom. Blood cultures typically grow colonizing bacteria from the gastrointestinal tract. The diagnosis can be confirmed with contrast-enhanced chest computed tomographic scan, which shows contrast migration from the left atrium toward the posterior mediastinum and esophagus or air inside the heart chambers. ${ }^{2}$ Any manipulation of the esophagus, including transesophageal echocardiography, should be avoided to prevent disruption of the fistulous trajectory. ${ }^{3}$ After confirmation of the presence of an AEF, urgent surgical exploration with primary closure of the hole in the posterior left atrium should follow, in which a pericardial flap over the esophagus can be used to close the posterior mediastinum.

In the applied thoracoscopic epicardial procedure, direct view of both the posterior left atrial wall and the esophagus was present, which allowed adequate spacing when applying clamps and rails. Concurrent fistula formation was therefore highly unexpected. AtriCure has, however, reported 8 other cases of AEF related to its Coolrail bipolar ablation rail.

In addition, we wonder whether the atrial septal occluder contributed to focal left atrial damage and thus fistula formation. The placement of the clamps to the right pulmonary veins most likely included the interatrial disc, which allowed the platinum left atrial distal marker band of the Amplatzer device to come into direct proximity with the posterior left atrial wall and serve as a thermal conductor, with focal tissue damage as result (Figure 1). We are not aware of a previously reported relationship between AEF 
and an atrial septal occluder, but a possible relationship thus may exist. Patients with atrial septum occluders are now excluded from our epicardial ablation program.

\section{References}

1. Dagres N, Kottkamp H, Piorkowski C, Doll N, Mohr F, Horlitz M, et al. Rapid detection and successful treatment of esophageal perforation after radiofre- quency ablation of atrial fibrillation. J Cardiovasc Electrophysiol. 2006;17: 1213-5.

2. Nair GM, Nery PB, Redpath CJ, Lam BK, Birnie DH. Atrioesophageal fistula in the era of atrial fibrillation ablation: a review. Can J Cardiol. 2014;30: 388-95.

3. Chevez P, Messerli FH, Casso Dominguez A, Aziz EF, Sichrovsky T, Garcia D et al. Atrioesophageal fistula following ablation procedures for atrial fibrillation: systematic review of case reports. Open Heart. 2015;2:e000257. 